\title{
BMJ Open Evidence on the effect of gender of newborn, antenatal care and postnatal care on breastfeeding practices in Ethiopia: a meta-analysis and meta-regression analysis of observational studies
}

Tesfa Dejenie Habtewold, ${ }^{\oplus, 2}$ Nigussie Tadesse Sharew, ${ }^{1}$ Sisay Mulugeta Alemu ${ }^{3}$

To cite: Habtewold TD, Sharew NT, Alemu SM. Evidence on the effect of gender of newborn, antenatal care and postnatal care on breastfeeding practices in Ethiopia: a metaanalysis andmeta-regression analysis of observational studies. BMJ Open 2019;9:e023956. doi:10.1136/ bmjopen-2018-023956

- Prepublication history and additional material for this paper are available online. To view these files, please visit the journal online (http://dx.doi. org/10.1136/bmjopen-2018023956).

Received 4 May 2018 Revised 1 April 2019 Accepted 17 April 2019
Check for updates

(C) Author(s) (or their employer(s)) 2019. Re-use permitted under CC BY-NC. No commercial re-use. See rights and permissions. Published by BMJ.

For numbered affiliations see end of article.

Correspondence to Tesfa Dejenie Habtewold; tesfadej2003@gmail.com

\section{ABSTRACT}

Objectives The aim of this systematic review and metaanalysis was to investigate the association of gender of newborn, antenatal care (ANC) and postnatal care (PNC) with timely initiation of breast feeding (TIBF) and exclusive breastfeeding (EBF) practices in Ethiopia.

Design Systematic review and meta-analysis.

Data sources To retrieve all available literature, PubMed, EMBASE, CINAHL, WHO Global Health Library, Web of Science and SCOPUS databases were systematically searched and complemented by manual searches. The search was done from August 2017 to September 2018.

Eligibility criteria All observational studies including crosssectional, case-control, cohort studies conducted in Ethiopia from 2000 to 2018 were included. Newcastle-Ottawa Scale was used for quality assessment of included studies.

Data extraction and synthesis Study area, design, population, number of mothers (calculated sample size and participated in the study) and observed frequency data were extracted using Joanna Briggs Institute tool. To obtain the pooled effect size, a meta-analysis using weighted inverse variance random-effects model was performed. Cochran's $Q X^{2}$ test, $\tau^{2}$ and $I^{2}$ statistics were used to test heterogeneity, estimate amount of total/ residual heterogeneity and measure variability attributed to heterogeneity, respectively. Mixed-effects meta-regression analysis was done to identify possible sources of heterogeneity. Egger's regression test at $p$ value threshold $\leq 0.01$ was used to examine publication bias. Furthermore, the trend of evidence over time was examined by performing a cumulative meta-analysis.

Results Of 523 articles retrieved, 17 studies ( $n=26146$ mothers) on TIBF and 24 studies ( $n=17819$ mothers) on EBF were included in the final analysis. ANC $(\mathrm{OR}=2.24$, $95 \% \mathrm{Cl} 1.65$ to $\left.3.04, \mathrm{p}<0.001, \mathrm{I}^{2}=90.9 \%\right)$, PNC (OR=1.86, $95 \% \mathrm{Cl} 1.41$ to $\left.2.47, \mathrm{p}<0.001, \mathrm{I}^{2}=63.4 \%\right)$ and gender of newborn $\left(\mathrm{OR}=1.31,95 \% \mathrm{Cl} 1.01\right.$ to $1.68, \mathrm{p}=0.04, \mathrm{I}^{2}=81.7 \%$ ) significantly associated with EBF. ANC $(\mathrm{OR}=1.70,95 \% \mathrm{Cl} 1.10$ to $2.65, p=0.02, l^{2}=93.1 \%$ ) was also significantly associated with TIBF but not with gender of newborn $(\mathrm{OR}=1.02,95 \% \mathrm{Cl}$ 0.86 to $1.21, p=0.82, I^{2}=66.2 \%$ ).
Strengths and limitations of this study

- This systematic review and meta-analysis was conducted based on the registered and published protocol.

- Since this is the first study in Ethiopia, the evidence could be helpful for future researchers, public health practitioners and healthcare policy-makers.

- Almost all included studies were observational which might weaken the strength of evidence and hinder causality inference.

- Perhaps, the results may not be nationally representative given that studies from some regions are lacking.

- Based on the conventional method of heterogeneity test, a few analyses suffer from high between-study variation.

Conclusions In line with our hypothesis, gender of newborn, ANC and PNC were significantly associated with EBF. Likewise, ANC was significantly associated with TIBF. Optimal care during pregnancy and after birth is important to ensure adequate breast feeding. This meta-analysis study provided up-to-date evidence on breastfeeding practices and its associated factors, which could be useful for breastfeeding improvement initiative in Ethiopia and cross-country and cross-cultural comparison.

Trial registration number CRD42017056768

\section{INTRODUCTION}

WHO and Unicef recommend timely initiation of breast feeding (TIBF) (ie, initiating breast feeding within 1 hour of birth) and exclusive breast feeding (EBF) (ie, feeding only human milk during the first 6 months $)^{1}$ for maintaining maternal and newborn health. ${ }^{2}$ Breast feeding provides optimal nutrition, increase cognitive development, reduce morbidity and mortality for the newborn; for 
example, TIBF prevents $22 \%$ of neonatal deaths. ${ }^{3}$ Inappropriate breastfeeding practice, on the other hand, causes more than two-thirds of under-five child mortality, of which $41 \%$ of these deaths occur in Sub-Saharan Africa. ${ }^{14}$ Breast feeding also prevents maternal long-term chronic diseases, such as diabetes mellitus. ${ }^{3}$

According to a new 2017 global Unicef and WHO report, only $42 \%$ start breast feeding within an hour of birth, leaving an estimated 78 million newborns to wait over 1 hour to be put to the breast, the majority born in low-income and middle-income countries. ${ }^{5}$ The prevalence rate of TIBF varies widely across regions from $35 \%$ in the Middle East and North Africa to $65 \%$ in Eastern and Southern Africa. Another report also shows that only two in five infants $<6$ months of age are exclusively breast fed. ${ }^{6}$ The prevalence rate of EBF ranges from $22 \%$ in East Asia and Pacific to $56 \%$ in Eastern and Southern Africa. ${ }^{6}$ Based on our meta-analysis in 2018, the prevalence of TIBF and EBF in Ethiopia is $66.5 \%$ and $60.1 \%$ respectively. ${ }^{7}$ To date, globally, only 22 nations have achieved the WHO goal of $70 \%$ coverage in TIBF and 23 countries have achieved at least $60 \%$ coverage in $\mathrm{EBF}^{2}$

To promote optimal breast feeding, WHO, Unicef and other (inter)national organisations have been working in developing countries, and several studies have been conducted on the advantages of breast feeding. However, it is still challenging to achieve the expected coverage and attributed to several factors including antenatal (ANC), postnatal care (PNC) and gender of newborn, ${ }^{89}$ and breastfeeding coverage continued to be suboptimal as a result. In Ethiopia, several meta-analyses studies were done on infant and young child feeding. ${ }^{70-14}$ In our previous meta-analysis, we explored the association between maternal employment, lactation counselling, mode of delivery, place of delivery, maternal age, newborn age and discarding colostrum breastfeeding practices (ie, TIBF and EBF) ${ }^{710}$ We also separately studied the association between TIBF and EBF. ${ }^{7}$ However, none of these meta-analyses did study the pooled effect of gender of newborn, ANC and PNC on TIBF and EBF. Given the absence of pooled estimates, up-to-date evidence is required to design intervention-based studies targeting these factors. Therefore, we aimed to investigate whether TIBF and EBF in Ethiopia are influenced by gender of newborn, ANC and PNC. We hypothesised at least one ANC or PNC visit significantly improves TIBF and EBF practices. Additionally, mothers with male newborn have higher odds of TIBF and EBF compared with mothers with female newborn.

\section{METHODS}

\section{Protocol registration and publication}

The study protocol was registered with the University of York, Centre for Reviews and Dissemination, International prospective register of systematic reviews (PROSPERO) and published. ${ }^{15}$

\section{Search strategy and databases}

PubMed, EMBASE, CINAHL, WHO Global Health Library, Web of Science and SCOPUS electronic databases were searched to extract all available literature. The search strategy was developed using Population Exposure Controls and Outcome (PECO) searching guide in consultation with a medical information specialist (online supplementary file 1). The search was done from August 2017 to September 2018. Grey literature and cross-references of included articles and previous meta-analysis were also hand searched.

\section{PECO guide}

Population

All mothers with newborn up to 23 months of age.

\section{Exposure}

Gender of the newborn, ANC and PNC visit (at least one visit).

\section{Comparison}

Female newborn, no ANC visit and no PNC visit.

\section{Outcome}

TIBF and EBF practices.

\section{Inclusion and exclusion criteria}

Studies were included if they met the following criteria: (1) observational studies including cross-sectional, case-control, cohort studies; (2) conducted in Ethiopia; (3) published in English language and (4) published between 2000 and 2018. Studies were excluded on any one of the following conditions: (1) conducted in women with HIV/AIDS, preterm newborn and newborn in intensive care unit; (2) published in language other than English; (3) abstracts without full text and (4) qualitative studies, symposium/conference proceedings, essays, commentaries and case reports.

\section{Selection and quality assessment}

Initially, all identified articles were exported to Refwork citation manager (RefWorks 2.0; ProQuest LLC, Bethesda, Maryland, USA, http://www.refworks.com), and duplicate studies were cancelled. Next, a pair of independent reviewers identified articles by analysing the title and abstract for relevance and its compliance with the proposed review topic. Agreement between the two reviewers, as measured by Cohen's Kappa ${ }^{16}{ }^{16}$ was 0.76 . After removing irrelevant studies through a respective decision after discussion, full texts were systematically reviewed for further eligibility analysis. Newcastle-Ottawa Scale (NOS) was used to examine the quality of studies and for potential risk of bias. ${ }^{17}$ In line with the WHO standard definition, outcome measurements were TIBF (the percentage of newborn who breast feed within the first hour of birth) and EBF (the percentage of infants who exclusively breast fed up to 6 months since birth). Finally, Joanna Briggs Institute (JBI) tool $^{18}$ was used to extract the following data: study area (region and place), method (design), population, number of mothers (calculated sample size and participated in 
the study) and observed data (ie, $2 \times 2$ table). Geographic regions were categorised based on the current Federal Democratic Republic of Ethiopia administrative structure. ${ }^{19}$ Disagreement between reviewers was solved through discussion and consensus.

\section{Statistical analysis}

A meta-analysis using a weighted inverse variance random-effects model was performed to obtain a pooled OR. In addition, a cumulative meta-analysis was done to illustrate the trend of evidence regarding the effect of gender of newborn, ANC and PNC on breastfeeding practices. Publication bias was assessed by visual inspection of a funnel plot and Egger's regression test for funnel plot asymmetry using $\mathrm{SE}$ as a predictor in mixed-effects meta-regression model at a $\mathrm{p}$ value threshold $\leq 0.010 .{ }^{20}$ Duval and Tweedie trim-and-fill method ${ }^{21}$ was used to manage publication bias. Cochran's $Q X^{2}$ test, $\tau^{2}$ and $I^{2}$ statistics were used to test heterogeneity, estimate amount of total/residual heterogeneity and measure variability attributed to heterogeneity, respectively. ${ }^{22}$ Mixed-effects meta-regression analysis was done to examine the effect of variation in study area (region), residence of women, sample size and publication year on between-study heterogeneity. ${ }^{23}$ The total amount of heterogeneity $\left(R^{2}\right)$ accounted for these factors was calculated by subtracting the residual amount of heterogeneity from the total amount of heterogeneity and dividing by the total amount of heterogeneity. Moreover, to assess the moderation effect of these factors, Omnibus test of moderators was applied. The data were analysed using 'metafor' packages in R software V.3.2.1 for Windows. ${ }^{23}$

\section{Data synthesis and reporting}

We analysed the data in two groups based on outcome measurements (ie, TIBF and EBF). Results are presented using forest plots. Preferred Reporting Items for Systematic Reviews and Meta-Analyses (PRISMA) guideline was strictly followed to report our results. ${ }^{24}$

\section{Minor post hoc protocol changes}

Based on the authors' decision and reviewers' recommendation, the following changes were made to our published protocol methods. ${ }^{15}$ We added the JBI tool ${ }^{18}$ to extract the data. In addition, we used the Duval and Tweedie trim-and-fill method to manage publication bias. Furthermore, cumulative meta-analysis and mixed-effects meta-regression analysis were done to reveal the trends of evidence and identify possible sources of between-study heterogeneity, respectively.

\section{Patient and public involvement}

The research questions and outcome measures were developed by the authors (TDH and NTS) in consultation with public health professionals and previous studies. Given this is a systematic review and meta-analysis based on published data, patients/study participants were not directly involved in the design and analysis of this study. The results of this study will be disseminated to patients/ study participants through health education on factors affecting breast feeding and disseminating the key findings using brochure in the local language.

\section{RESULTS}

\section{Search results}

In total, we obtained 533 articles from PubMed $(n=169)$, EMBASE $(n=24)$, Web of Science $(n=200)$, SCOPUS $(\mathrm{n}=85)$ and CINHAL and WHO Global Health Library $(n=5)$. Fifty additional articles were found through manual search. After removing duplicates and screening of titles and abstracts, 84 studies were selected for fulltext review. Of these, 43 articles were excluded due to several reasons: 19 studies on complementary feeding, 3 studies on prelacteal feeding, 3 studies on malnutrition, 17 studies with different variables of interest and 1 project review report. As a result, 41 articles fulfilled the inclusion criteria and used in this meta-analysis: 17 studies investigated the association between TIBF and gender of newborn and ANC whereas 24 studies between EBF and gender of newborn, ANC and PNC. The PRISMA flow diagram of literature screening and selection process is shown in figure 1 . One study could report more than one outcome measures or associated factors.

\section{Study characteristics}

As presented in table 1, 17 studies reported the association of TIBF and gender of newborn and ANC in 26146 mothers. Among these studies, 13 of them were conducted in Amhara $(n=5)$, Oromia $(n=4)$ and Southern Nations, Nationalities and Peoples' (SNNP) $(n=4)$ region. Regarding the residence status, eight studies were conducted in both urban and rural whereas six studies in urban women. All studies passed the NOS quality assessment criteria at a cut-off value $\geq 7$.

Twenty-four studies reported the association between EBF and gender of newborn, ANC and PNC in 17819 mothers. Of these studies, 11 were conducted in Amhara and seven in SNNP region. Based on the residence status, 10 studies were conducted in urban, 8 in urban and rural, and 6 in rural women. Even though almost all studies were cross-sectional, five studies have used nationally representative data of the Ethiopian Demographic Health Survey. ${ }^{19-23}$ Detailed characteristics of the included studies are shown in table 2.

\section{Meta-analysis}

Timely initiation of breast feeding

Among the 17 selected studies, 10 studies ${ }^{25-34}$ reported the association between TIBF and gender of newborn in 16411 mothers (table 1A). The pooled OR of gender of newborn was 1.02 ( $95 \%$ CI 0.86 to $1.21, \mathrm{p}=0.82, \mathrm{I}^{2}=66.2 \%$ ) (figure 2). Mothers with male newborn had 2\% higher chance of initiating breast feeding within 1 hour of birth compared with female newborn although not statistically significant. There was no significant publication bias $(\mathrm{z}=0.41, \mathrm{p}=0.68)$ (online supplementary figure 1$)$. 


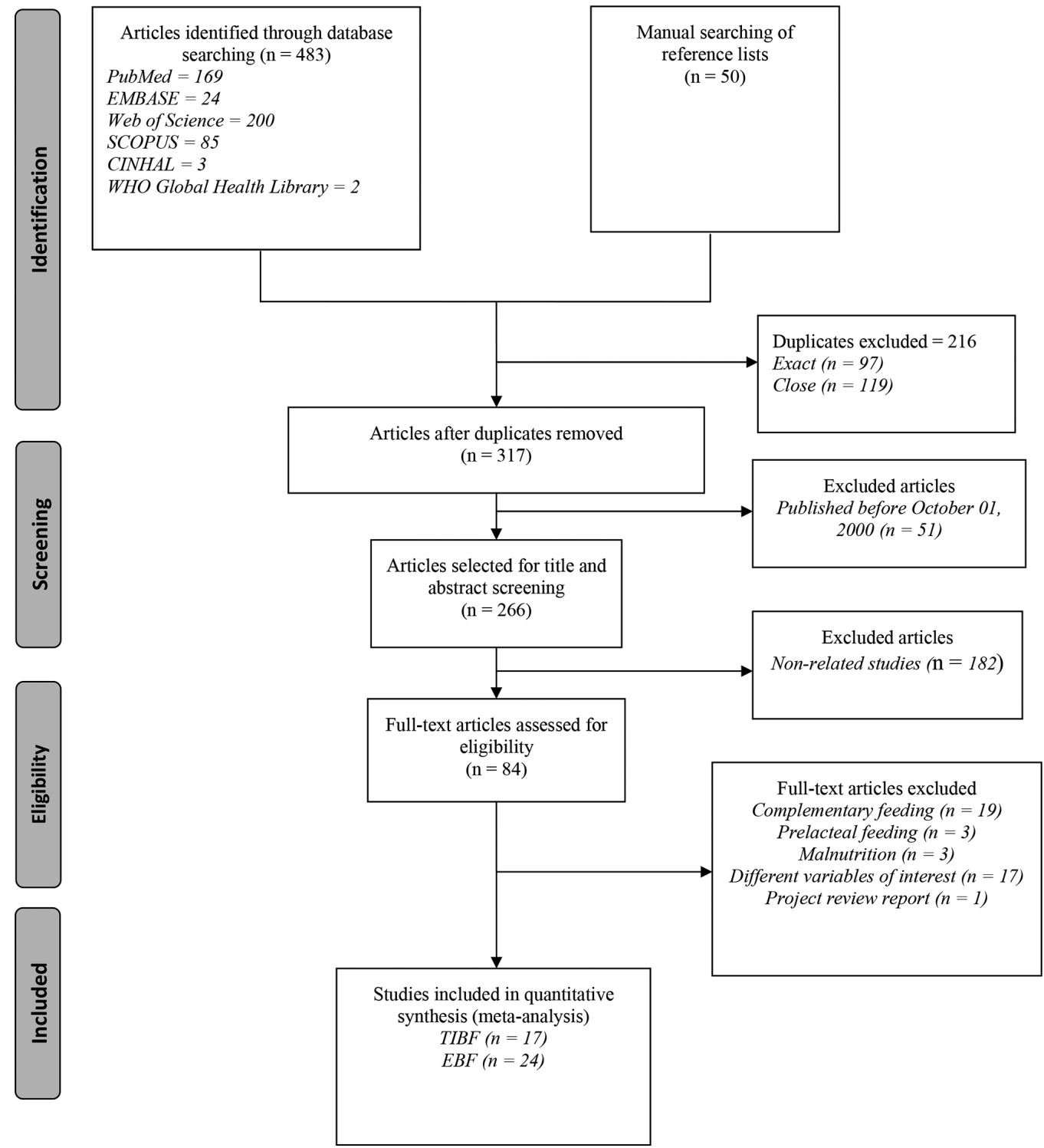

Figure 1 PRISMA flow diagram of literature screening and selection process; ' $n$ ' in each stage represents the total number of studies that fulfilled particular criteria. EBF, exclusive breast feeding; PRISMA, Preferred Reporting Items for Systematic Reviews and Meta-Analyses; TIBF, timely initiation of breast feeding.

Likewise, 13 studies $^{27} 283031$ 33-41 reported the association between TIBF and ANC in 12535 mothers (table 1B). The pooled OR of ANC was 1.70 (95\% CI 1.10 to 2.65, $\mathrm{p}=0.02, \mathrm{I}^{2}=93.1 \%$ ) (figure 3 ). Mothers who had at least one ANC visit had $70 \%$ significantly higher chance of initiating breast feeding within 1 hour of birth compared with mothers who had no ANC visit. There was no significant publication bias $(\mathrm{z}=0.96, \mathrm{p}=0.34)$ (online supplementary figure 2).

\section{Exclusive breast feeding}

Out of the 24 studies included, 11 studies ${ }^{25} 26$ 42-50 reported the association between $\mathrm{EBF}$ and gender of newborn in 6527 mothers (table 2A). The pooled OR of newborn gender was 1.08 (95\% CI 0.86 to $1.36, \mathrm{p}=0.49$, $\mathrm{I}^{2}=71.7 \%$ ) (figure 4 ). Since significant publication bias detected $(\mathrm{z}=-3.64, \mathrm{p}<0.001)$, we did Duval and Tweedie trim-and-fill analysis and calculated a new effect size for gender of newborn $(\mathrm{OR}=1.31,95 \%$ CI 1.01 to 1.68 , $\mathrm{p}=0.04, \quad \mathrm{I}^{2}=81.7 \%$ ) after including imputed studies (ie, estimated number of missing studies $=4$ ) (online supplementary figure 3 ). Therefore, mothers with male newborn had $31 \%$ significantly higher chance of exclusive breast feeding during the first 6 months compared with mothers with female newborn.

Twenty-one studies ${ }^{35-37} 42-4951-60$ reported the association between EBF and ANC in 16052 mothers (table 2B). The pooled OR of ANC was 2.24 (95\% CI 1.65 to $3.04, \mathrm{p}<0.0001, \mathrm{I}^{2}=90.9 \%$ ) (figure 5 ). Mothers who had at least one ANC visit had 2.24 times significantly higher chance of exclusively breast feed compared with mothers who had no ANC visit. There was no significant publication bias $(\mathrm{z}=1.69, \mathrm{p}=0.09)$ (online supplementary figure 4). 


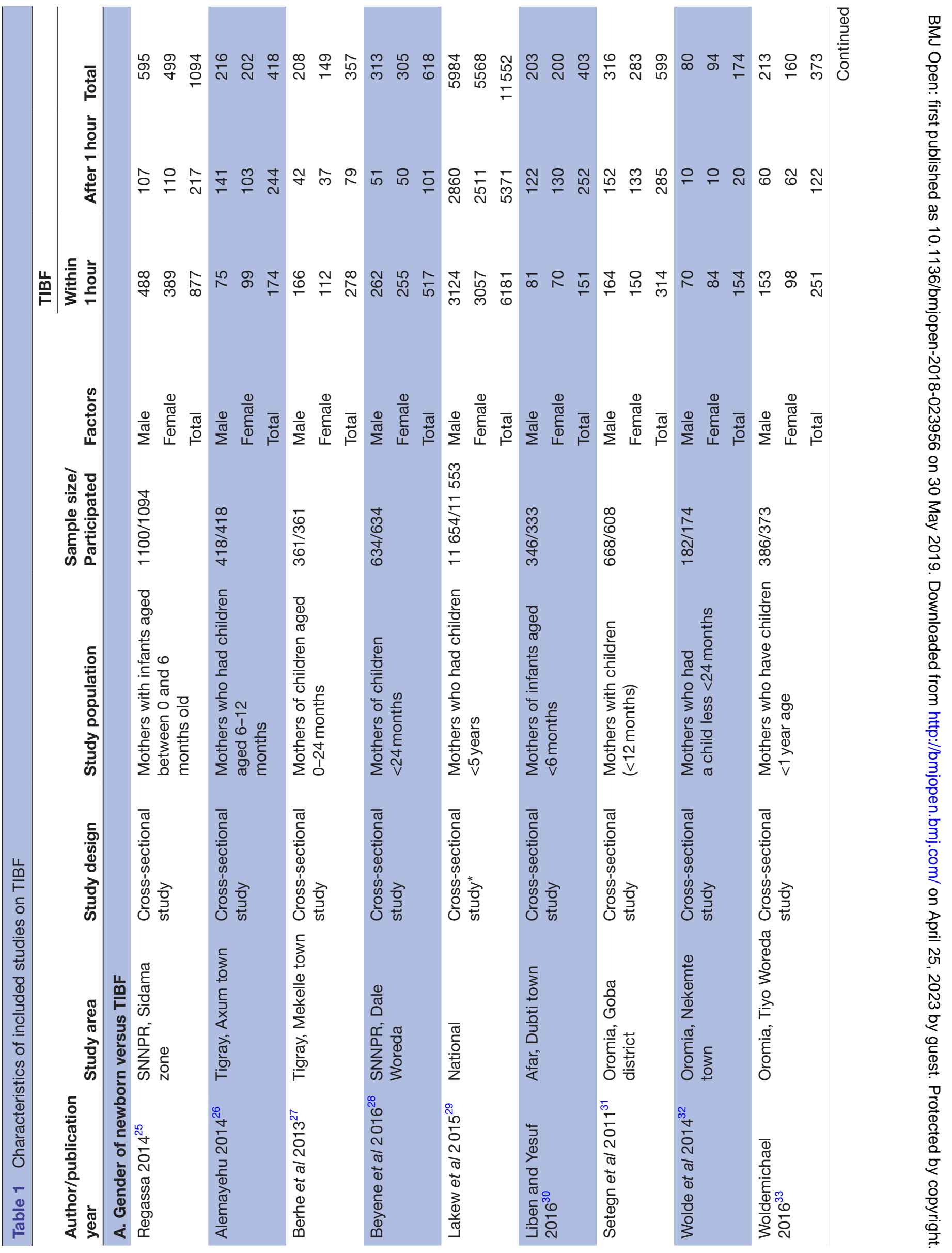




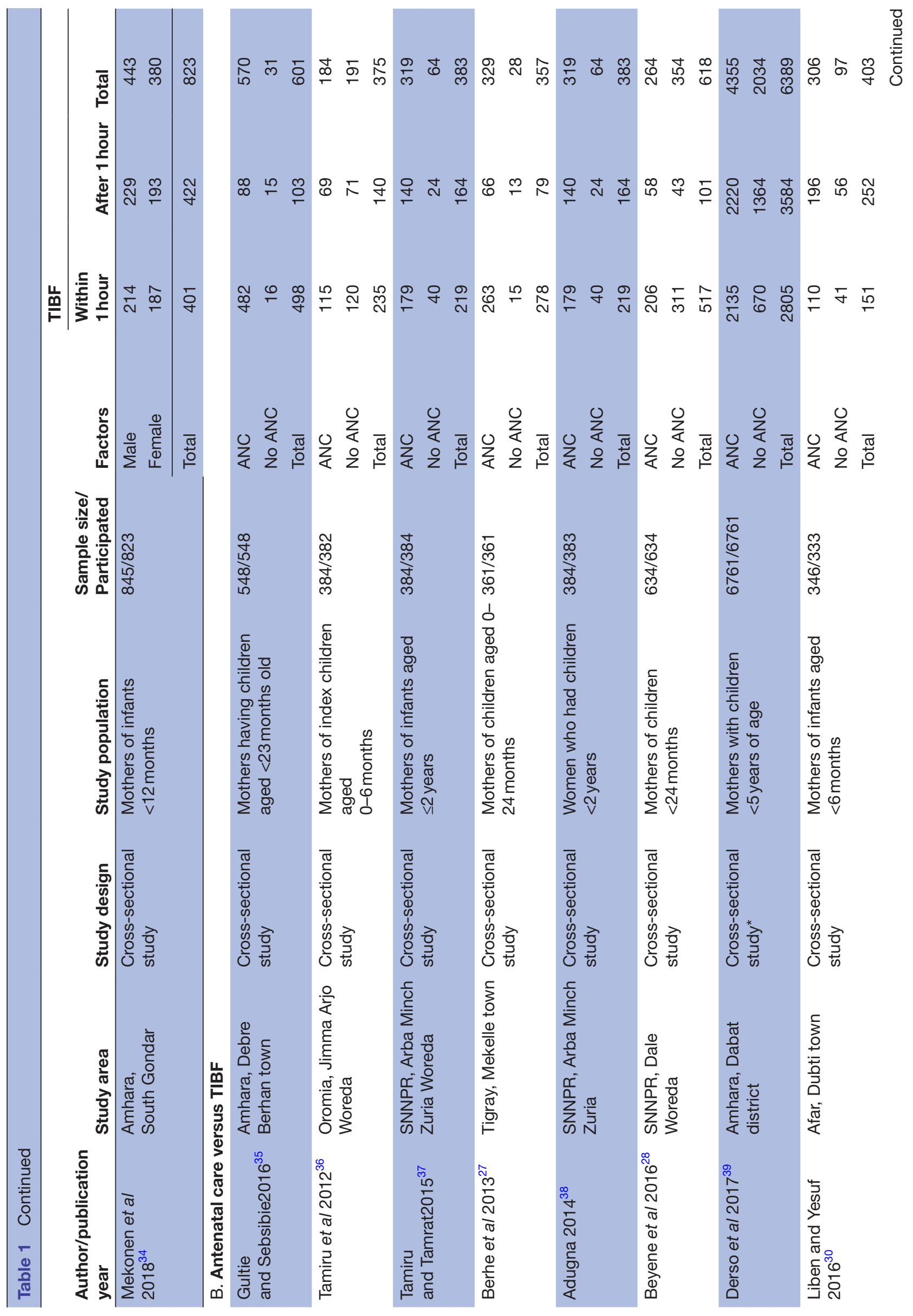




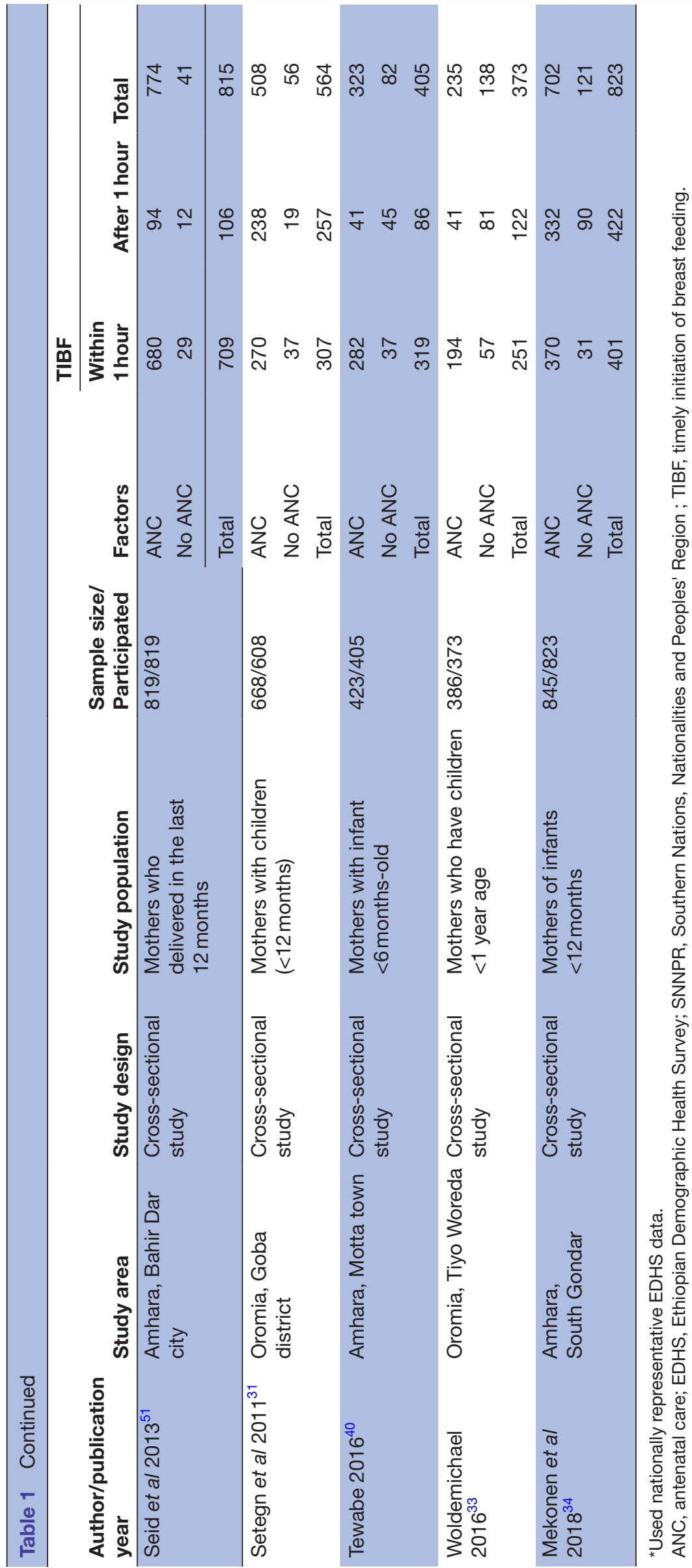

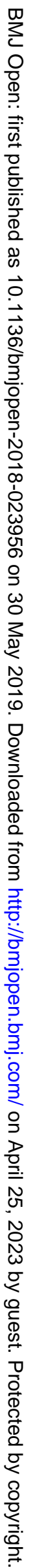




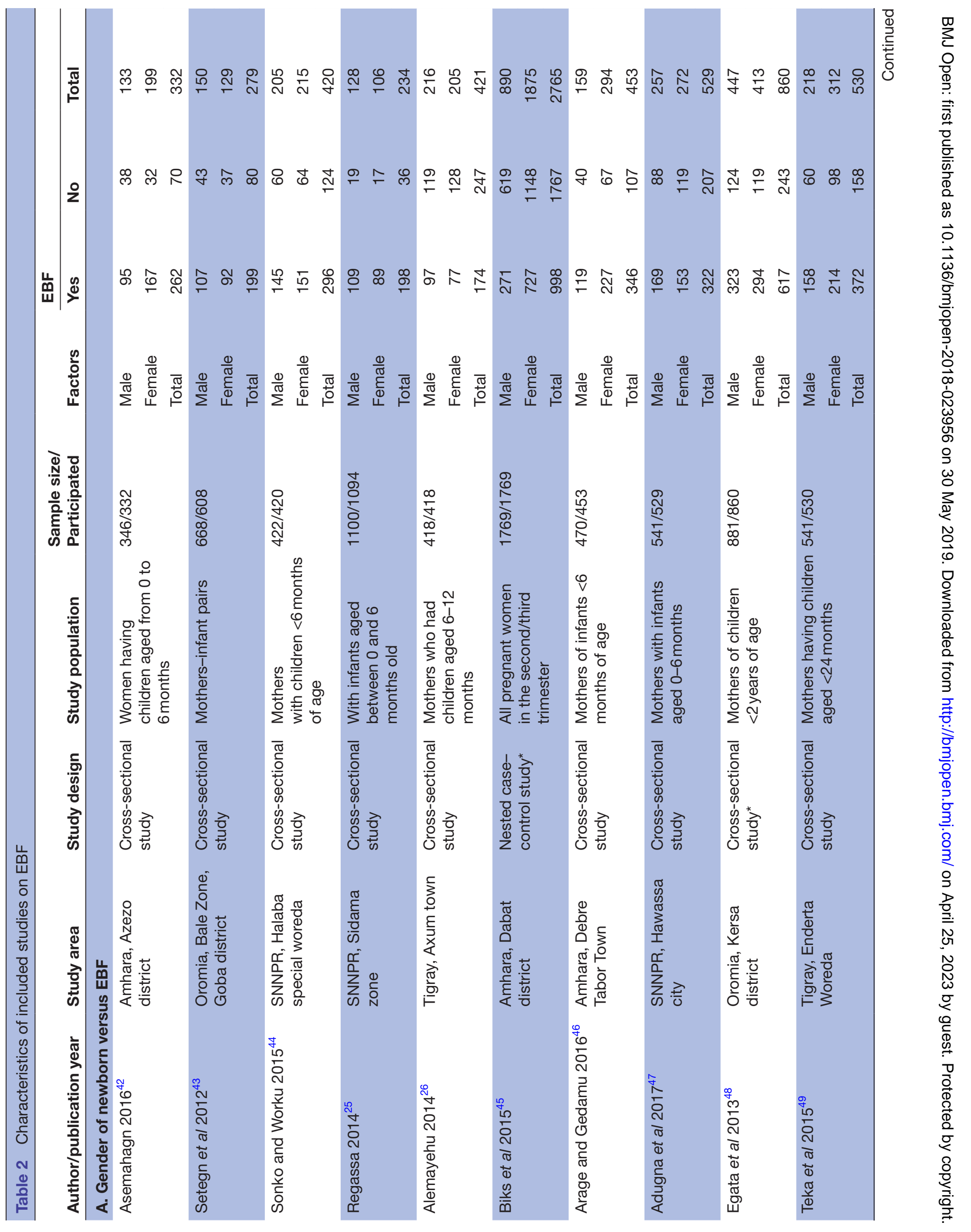




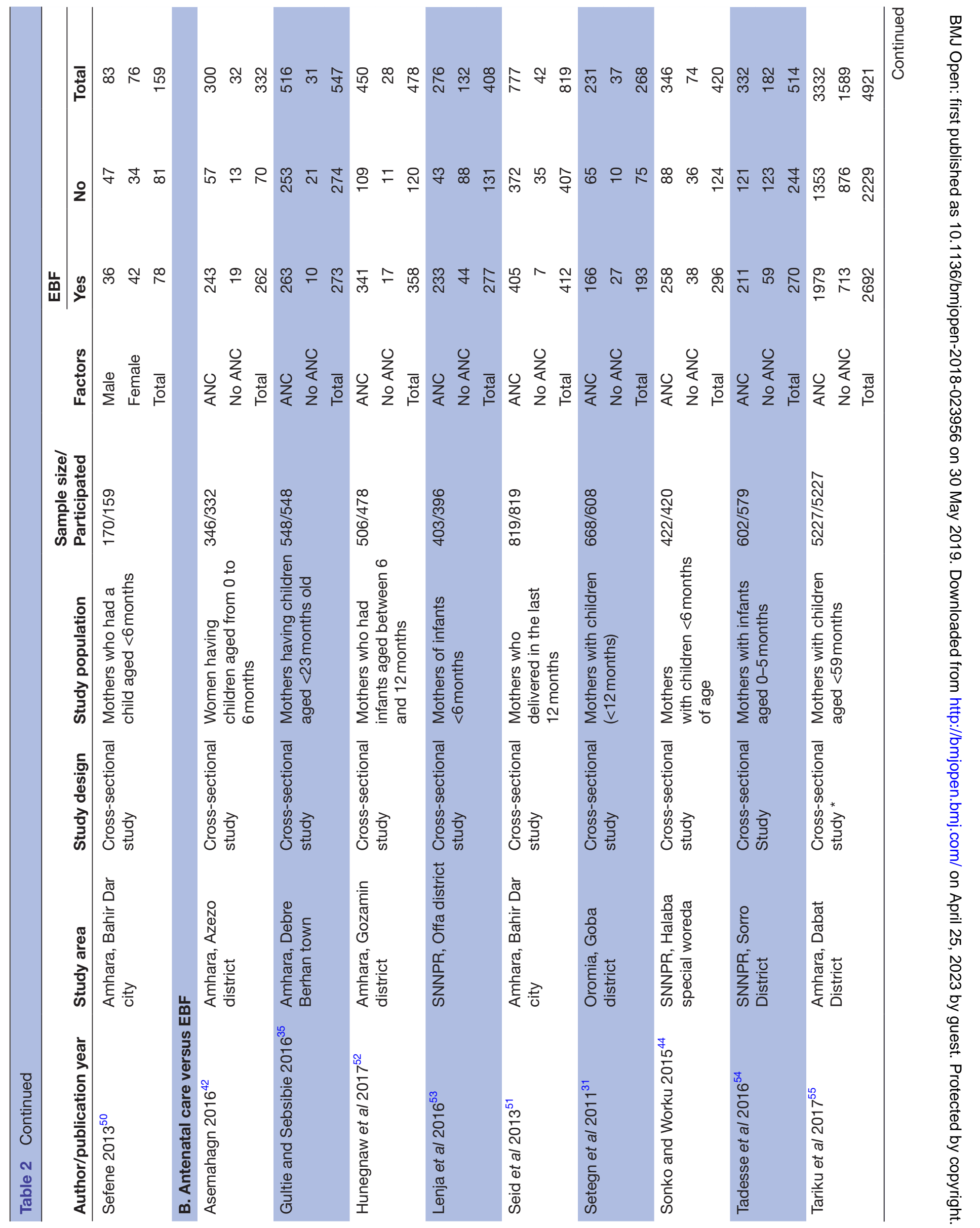




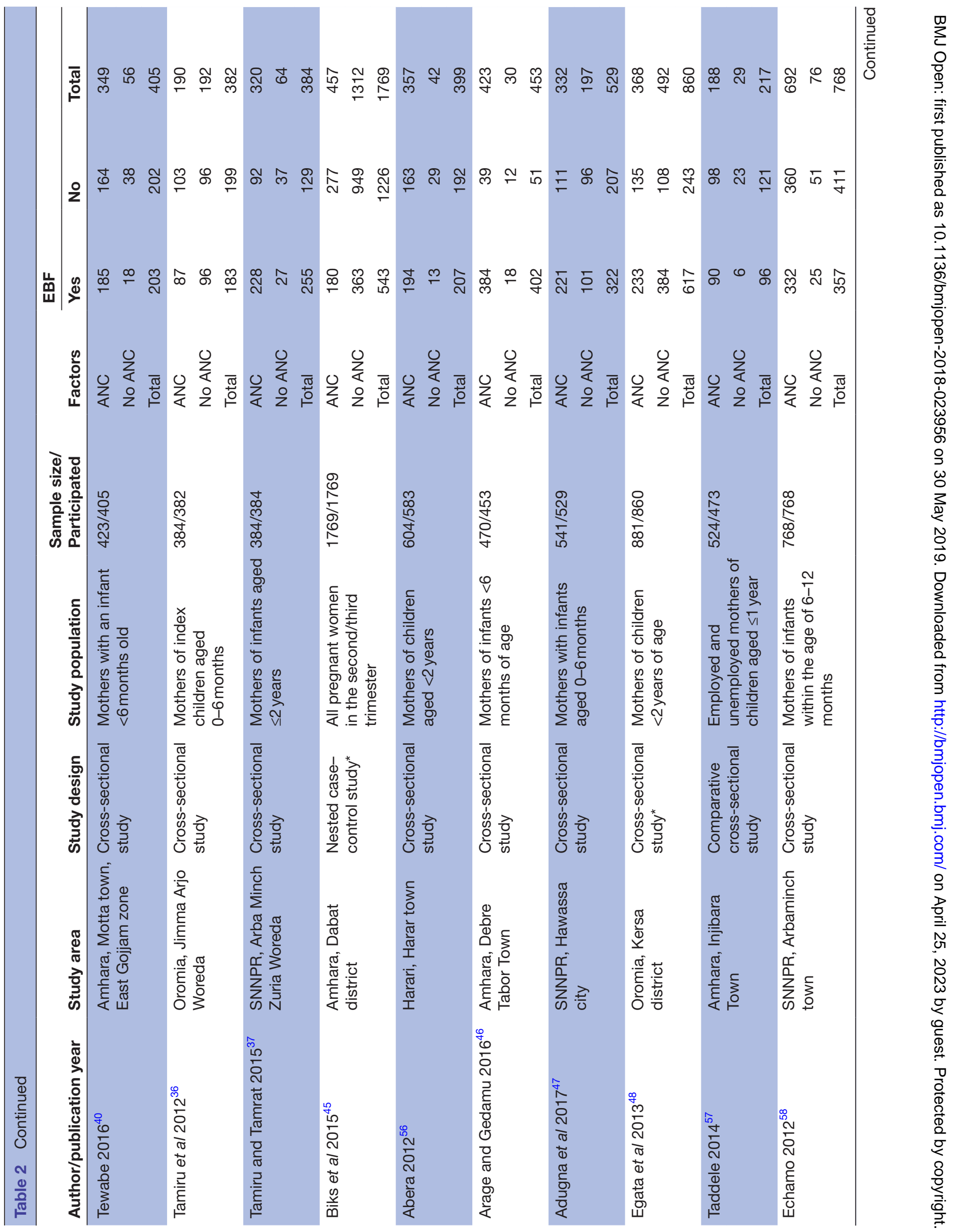




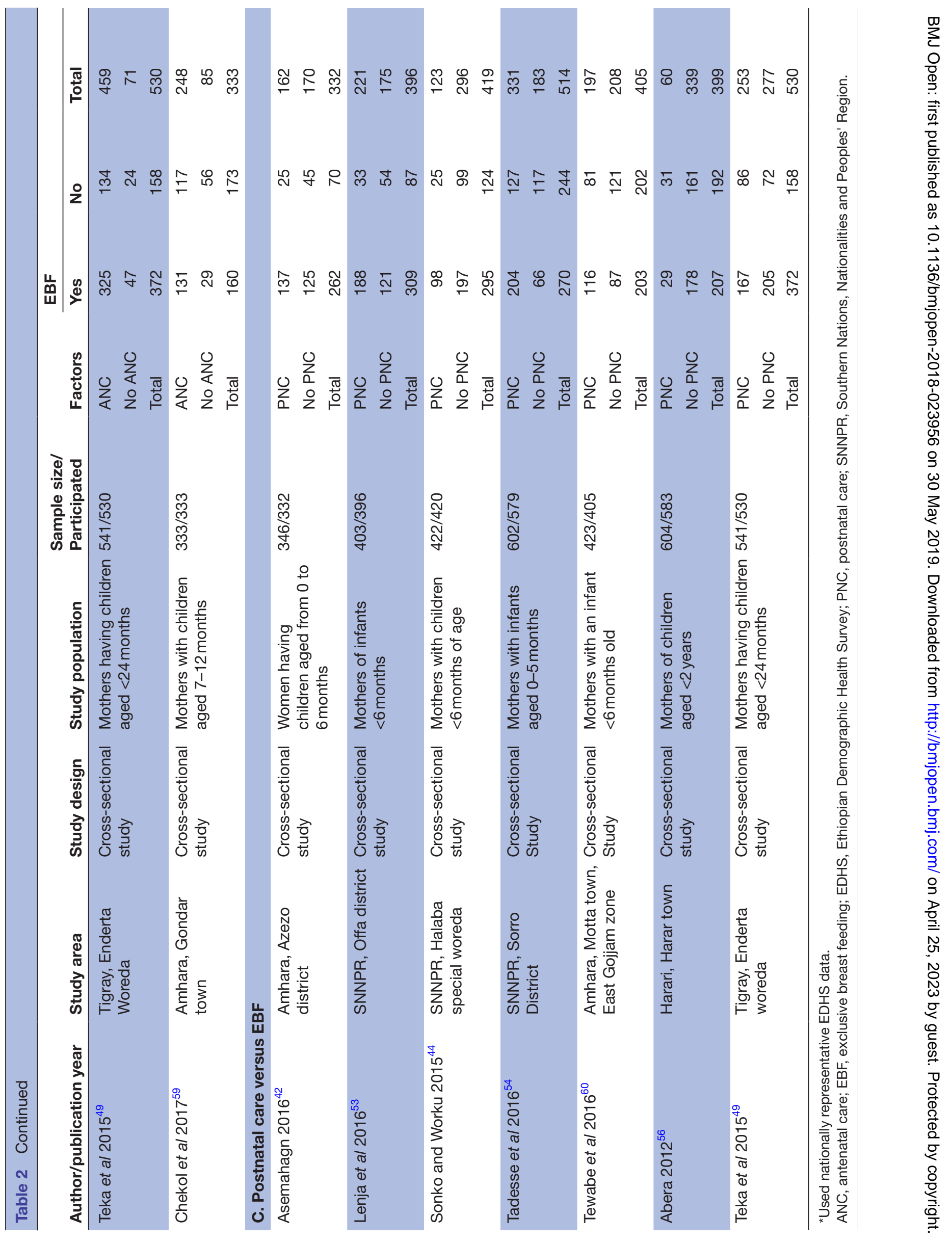




\begin{tabular}{|c|c|c|c|c|c|}
\hline \multirow[b]{2}{*}{ Studies and Publication year } & \multicolumn{2}{|c|}{ Male } & \multicolumn{2}{|c|}{ Female } & \multirow[b]{2}{*}{ Odds Ratio $[95 \% \mathrm{Cl}]$} \\
\hline & TIBF & LIBF & TIBF & LIBF & \\
\hline Regassa; 2014 & 488 & 107 & 389 & 110 & $1.29[0.96,1.74]$ \\
\hline Alemayehu et al,; 2014 & 75 & 141 & 99 & 103 & $0.55[0.37,0.82]$ \\
\hline Berhe et al.; 2013 & 166 & 42 & 112 & 37 & - $1.31[0.79,2.16]$ \\
\hline Beyene et al.; 2017 & 262 & 51 & 255 & 50 & $1.01[0.66,1.54]$ \\
\hline Lakew et al; 2015 & 3124 & 2860 & 3057 & 2511 & $0.90[0.83,0.97]$ \\
\hline Liben et al; 2016 & 81 & 122 & 70 & 130 & $1.23[0.82,1.85]$ \\
\hline Setegn et al; 2011 & 164 & 152 & 150 & 133 & $0.96[0.69,1.32]$ \\
\hline Wolde et al; 2014 & 70 & 10 & 84 & 10 & $0.83[0.33,2.12]$ \\
\hline Woldemichael et al; 2016 & 153 & 60 & 98 & 62 & - $1.61[1.04,2.50]$ \\
\hline Mekonen et al; 2018 & 214 & 229 & 187 & 193 & $0.96[0.73,1.27]$ \\
\hline \multicolumn{5}{|c|}{ Summary } & $1.02[0.86,1.21]$ \\
\hline REM test for heterogeneity $(\mathrm{Q}=$ & $22.28, d$ & If $=9, p$ & $01 ; 1^{2}=$ & $66.2 \%)$ & \\
\hline
\end{tabular}

Favours LIBF Favours TIBF

Figure 2 Forest plot of the unadjusted odds ratios with corresponding $95 \%$ Cls of 10 studies on the association of gender of newborn and TIBF. The horizontal line represents the $\mathrm{Cl}$, the box and its size in the middle of the horizontal line represents the weight of sample size. The polygon represents the pooled OR. The reference category is 'Female'. LIBF, late initiation of breast feeding; REM, random-effects model; TIBF, timely initiation of breast feeding.

Furthermore, seven studies ${ }^{42} 444953545660$ reported the association between EBF and PNC in 2995 mothers (table 2C). The pooled OR of PNC was 1.86 (95\% CI 1.41 to $2.47, \mathrm{p}<0.0001, \mathrm{I}^{2}=63.4 \%$ ) (figure 6 ). Mothers

\begin{tabular}{|c|c|c|c|c|c|c|}
\hline \multicolumn{3}{|c|}{ ANC } & \multicolumn{2}{|c|}{ No ANC } & \multirow{2}{*}{\multicolumn{2}{|c|}{ Odds Ratio $[95 \% \mathrm{Cl}]$}} \\
\hline Studies and Publication year & TIBF & LIBF & TIBF & LIBF & & \\
\hline Gultie et al; 2016 & 428 & 88 & 16 & 15 & $\longmapsto$ & $4.56[2.17,9.56]$ \\
\hline Tamiru et al.; 2012 & 115 & 69 & 120 & 71 & & $0.99[0.65,1.50]$ \\
\hline Tamiru et al; 2015 & 179 & 140 & 40 & 24 & & $0.77[0.44,1.33]$ \\
\hline Berhe et al.; 2013 & 263 & 66 & 15 & 13 & 一 & $3.45[1.57,7.61]$ \\
\hline Adugna; 2014 & 179 & 140 & 40 & 24 & & $0.77[0.44,1.33]$ \\
\hline Beyene et al.; 2017 & 206 & 58 & 311 & 43 & & $0.49[0.32,0.76]$ \\
\hline Derso et al.; 2017 & 2135 & 2220 & 670 & 1364 & - & $1.96[1.75,2.19]$ \\
\hline Liben et al; 2016 & 110 & 196 & 41 & 56 & & $0.77[0.48,1.22]$ \\
\hline Seid; 2014 & 680 & 94 & 29 & 12 & $\longrightarrow$ & $2.99[1.48,6.07]$ \\
\hline Setegn et al; 2011 & 270 & 238 & 37 & 45 & - & $1.38[0.86,2.20]$ \\
\hline Tewabe; 2016 & 282 & 67 & 37 & 19 & 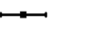 & $2.16[1.17,3.99]$ \\
\hline Woldemichael et al; 2016 & 194 & 41 & 57 & 81 & $\mapsto$ & $6.72[4.17,10.84]$ \\
\hline Mekonen et al; 2018 & 370 & 332 & 31 & 90 & $\rightarrow$ & $3.24[2.10,4.99]$ \\
\hline $\begin{array}{l}\text { Summary } \\
\text { REM test for heterogeneity ( } C\end{array}$ & & & & $3.1 \%)$ & & $1.70[1.10,2.65]$ \\
\hline
\end{tabular}

$$
\begin{array}{llll}
0.08 & 1 & 3 & 11 \\
\text { Favours LIBF } & \text { Favours TIBF }
\end{array}
$$

Figure 3 Forest plot of the unadjusted odds ratios with corresponding $95 \%$ Cls of 13 studies on the association of ANC and TIBF. The horizontal line represents the $\mathrm{Cl}$, the box and its size in the middle of the horizontal line represents the weight of sample size. The polygon represents the pooled OR. The reference category is 'No ANC follow-up'. ANC, antenatal care; LIBF, late initiation of breast feeding; REM,

\begin{tabular}{|c|c|c|c|c|c|}
\hline \multirow[b]{2}{*}{ Studies and Publication year } & \multicolumn{2}{|c|}{ Male } & \multicolumn{2}{|c|}{ Female } & \multirow[b]{2}{*}{ Odds Ratio $[95 \% \mathrm{Cl}]$} \\
\hline & EBF & NEBF & EBF & NEBF & \\
\hline Asemahagn; 2016 & 95 & 38 & 167 & 32 & $0.48[0.28,0.82]$ \\
\hline Setegn et al.; 2012 & 107 & 43 & 92 & 37 & $1.00[0.59,1.68]$ \\
\hline Sonko et al; 2015 & 145 & 60 & 151 & 64 & $1.02[0.67,1.56]$ \\
\hline Regassa; 2014 & 109 & 19 & 89 & 17 & $1.10[0.54,2.23]$ \\
\hline Alemayehu et al,; 2014 & 97 & 119 & 77 & 128 & $1.36[0.92,2.00]$ \\
\hline Biks et al; 2015 & 271 & 619 & 272 & 1148 & $\Rightarrow \quad 1.85[1.52,2.24]$ \\
\hline Arage et al; 2016 & 119 & 40 & 227 & 67 & $0.88[0.56,1.38]$ \\
\hline Adugna et al; 2017 & 169 & 88 & 153 & 119 & $1.49[1.05,2.12]$ \\
\hline Egata et al; 2013 & 323 & 124 & 294 & 119 & $1.05[0.78,1.42]$ \\
\hline Teka et al; 2015 & 158 & 60 & 214 & 98 & $1.21[0.82,1.77]$ \\
\hline Sefene et al; 2013 & 36 & 47 & 42 & 34 & $0.62[0.33,1.16]$ \\
\hline \multicolumn{5}{|c|}{$\begin{array}{l}\text { Summary } \\
\text { REM test for heterogeneity ( } Q=39.19, \mathrm{df}=10, p=0.00 ; I^{2}=71.7 \% \text { ) }\end{array}$} & $1.08[0.86,1.36]$ \\
\hline
\end{tabular}
random-effects model; TIBF, timely initiation of breast feeding.
Favours NEBF Favours EBF

Figure 4 Forest plot of the unadjusted odds ratios with corresponding $95 \%$ Cls of 11 studies on the association of newborn gender and EBF. The horizontal line represents the $\mathrm{Cl}$, the box and its size in the middle of the horizontal line represents the weight of sample size. The polygon represents the pooled OR. The reference category is 'Female'. EBF, exclusive breast feeding; NEBF, non exclusive of breast feeding; REM, random-effects model.

who had at least one PNC visit had $86 \%$ significantly higher chance of exclusively breast feed during the

\begin{tabular}{|c|c|c|c|c|c|c|}
\hline Studies and Publication year & $\begin{array}{l}\text { AN } \\
\text { EBF }\end{array}$ & NEBF & $\begin{array}{l}\text { No } \\
\text { EBF }\end{array}$ & $\begin{array}{l}\text { ANC } \\
\text { NEBF }\end{array}$ & & Odds Ratio $[95 \% \mathrm{Cl}]$ \\
\hline Asemahagn; 2016 & 243 & 57 & 19 & 13 & $\longrightarrow$ & $2.92[1.36,6.25]$ \\
\hline Gultie et al; 2016 & 263 & 253 & 10 & 21 & & $2.18[1.01,4.73]$ \\
\hline Hunegnawu et al; 2017 & 341 & 109 & 17 & 11 & & $2.02[0.92,4.45]$ \\
\hline Lenja et al.: 2016 & 223 & 43 & 44 & 88 & & $-10.37[6.37,16.89]$ \\
\hline Seid et al; 2013 & 405 & 372 & 7 & 35 & & 一 $\quad 5.44[2.39,12.40]$ \\
\hline Setegn et al.; 2012 & 166 & 65 & 27 & 10 & & $0.95[0.43,2.06]$ \\
\hline Sonko et al; 2015 & 258 & 88 & 38 & 36 & $=$ & $2.78[1.66,4.65]$ \\
\hline Tadesse et al; 2016 & 211 & 121 & 59 & 123 & & $3.64[2.48,5.33]$ \\
\hline Tariku et al.; 2017 & 1979 & 1353 & 713 & 876 & & $1.80[1.59,2.03]$ \\
\hline Tewabe et al.; 2017 & 185 & 164 & 18 & 38 & & $2.38[1.31,4.33]$ \\
\hline Tamiru et al.; 2012 & 87 & 103 & 96 & 96 & & $0.84[0.57,1.26]$ \\
\hline Tamiru et al; 2015 & 228 & 92 & 27 & 37 & $\rightarrow$ & $3.40[1.96,5.90]$ \\
\hline Biks et al; 2015 & 180 & 277 & 363 & 949 & & $1.70[1.36,2.12]$ \\
\hline Abera; 2012 & 194 & 163 & 13 & 29 & & $2.66[1.34,5.28]$ \\
\hline Arage et al; 2016 & 384 & 39 & 18 & 12 & & $6.56[2.95,14.63]$ \\
\hline Adugna et al; 2017 & 221 & 111 & 101 & 96 & $\rightarrow$ & $1.89[1.32,2.71]$ \\
\hline Egata et al; 2013 & 233 & 135 & 384 & 108 & & $0.49[0.36,0.66]$ \\
\hline Taddele et al; 2014 & 90 & 98 & 6 & 23 & & $3.52[1.37,9.04]$ \\
\hline Echamo; 2012 & 332 & 360 & 25 & 51 & & $1.88[1.14,3.11]$ \\
\hline Teka et al; 2015 & 325 & 134 & 47 & 24 & & $1.24[0.73,2.11]$ \\
\hline Chekol et al; 2017 & 131 & 117 & 29 & 56 & & $2.16[1.29,3.61]$ \\
\hline \multicolumn{5}{|c|}{$\begin{array}{l}\text { Summary } \\
\text { REM test for heterogeneity }\left(Q=185.02, \mathrm{df}=20, p=0.00 ; I^{2}=90.9 \%\right)\end{array}$} & - & $2.24[1.65,3.04]$ \\
\hline & & & & 0.08 & 3 & 17 \\
\hline \multicolumn{5}{|r|}{ Favours NEBF } & \multicolumn{2}{|c|}{ Favours EBF } \\
\hline
\end{tabular}
first 6 months compared with mothers who had no PNC

Figure 5 Forest plot of the unadjusted odds ratios with corresponding $95 \%$ Cls of 21 studies on the association of ANC and EBF. The horizontal line represents the $\mathrm{Cl}$, the box and its size in the middle of the horizontal line represents the weight of sample size. The polygon represents the pooled OR. The reference category is 'No ANC follow-up'. ANC, antenatal care; EBF, exclusive breast feeding; NEBF, nonexclusive of breast feeding; REM, random-effects model. 
PNC No PNC

Studies and Publication year EBF NEBF EBF NEB

Odds Ratio $[95 \% \mathrm{Cl}]$

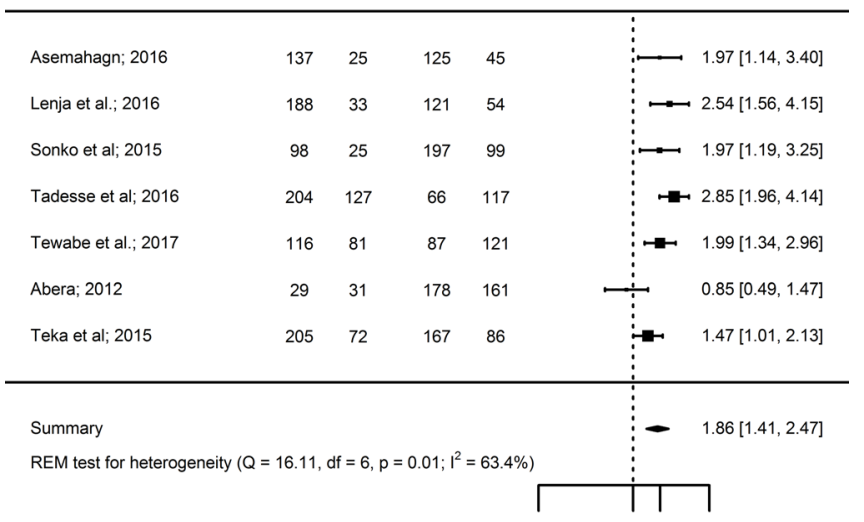

$0.09 \quad 127$

Favours NEBF Favours EBF

Figure 6 Forest plot of the unadjusted odds ratios with corresponding $95 \% \mathrm{Cls}$ of seven studies on the association of PNC and EBF. The horizontal line represents the $\mathrm{Cl}$, the box and its size in the middle of the horizontal line represents the weight of sample size. The polygon represents the pooled OR. The reference category is 'No PNC follow-up'. EBF, exclusive breast feeding; NEBF, non-exclusive breast feeding; PNC, postnatal care; REM, random-effects model.

follow-up. There was no significant publication bias $(\mathrm{z}=-0.91, \mathrm{p}=0.36)$ (online supplementary figure 5).

\section{Cumulative meta-analysis}

As illustrated in figure 7, the effect of gender of newborn (figure 7) has not been changed whereas the effect of ANC on TIBF (figure 8) has been increasing over time.

Similarly, the effect of gender of newborn on EBF (figure 9) has not been changed over time. The effect

Setegn et al; 2011
+ Berhe et al.; 2013
+ Regassa; 2014
+ Alemayehu et al,; 2014
+ Wolde et al; 2014
+ Lakew et al; 2015
+ Liben et al; 2016
+ Woldemichael et al; 2016
+ Beyene et al.; 2017
+ Mekonen et al; 2018

Odds Ratio (log scale)

Figure 7 Forest plot showing the results from a cumulative meta-analysis of studies examining the effect of gender of newborn on TIBF. TIBF, timely initiation of breast feeding.

Setegn et al; 2011
+ Tamiru et al.; 2012
+ Berhe et al.; 2013
+ Adugna; 2014
+ Seid; 2014
+ Tamiru et al; 2015
+ Gultie et al; 2016
+ Liben et al; 2016
+ Tewabe; 2016
+ Woldemichael et al; 2016
+ Beyene et al.; 2017
+ Derso et al.; 2017
+ Mekonen et al; 2018

$1.38[0.86,2.20]$

$1.15[0.83,1.59]$

$1.56[0.80,3.05]$

$1.30[0.74,2.30]$

$1.53[0.87,2.66]$

$1.35[0.82,2.23]$

$1.61[0.93,2.76]$

$1.45[0.88,2.39]$

$1.51[0.97,2.37]$

$1.79[1.08,2.97]$

$1.58[0.95,2.65]$

$1.61[1.01,2.57]$

$1.70[1.10,2.65]$

$\begin{array}{llll}0.5 & 1 & 2 & 3.5\end{array}$

Odds Ratio (log scale)

Figure 8 Forest plot showing the results from a cumulative meta-analysis of studies examining the effect of ANC on TIBF. ANC, antenatal care; TIBF, timely initiation of breast feeding.

of ANC (figure 10) and PNC (figure 11) have been increasing.

\section{Meta-regression analysis}

In studies reporting the association between TIBF and ANC, 26.29\% of the heterogeneity was accounted for the variation in study area (region), residence of mothers, sample size and publication year. Based on the omnibus test of moderators, however, none of these factors influenced association between TIBF and ANC $\left(Q_{M}=11.57\right.$, $\mathrm{df}=8, \mathrm{p}=0.17$ ). In studies reporting the association between TIBF and gender of newborn, the estimated amount of total heterogeneity was substantially low $\left(\operatorname{tau}^{2}=4.28 \%\right)$;

Studies and Publication year

Odds ratio $[95 \% \mathrm{Cl}]$

Setegn et al.; 2012
+ Egata et al; 2013
+ Sefene et al; 2013
+ Regassa; 2014
+ Alemayehu et al,; 2014
+ Sonko et al; 2015
+ Biks et al; 2015
+ Teka et al; 2015
+ Asemahagn; 2016
+ Arage et al; 2016
+ Adugna et al; 2017

Odds Ratio (log scale)

Figure 9 Forest plot showing the results from a cumulative meta-analysis of studies examining the effect of gender of newborn on EBF. EBF, exclusive breast feeding. 
Studies and Publication year

Setegn et al.; 2012

+ Tamiru et al.; 2012

+ Abera; 2012

+ Echamo; 2012

+ Egata et al; 2013
+ Taddele et al; 2014

+ Sonko et al; 2015

+ Tamiru et al; 2015

+ Biks et al; 2015

+ Teka et al; 2015

+ Gultie et al; 2016

+ Lenja et al.; 2016

+ Tadesse et al; 2016

+ Arage et al; 2016

+ Tariku et al.; 2017

+ Chekol et al; 2017
Seid et al; 2013

+ Asemahagn; 2016

+ Hunegnawu et al; 2017

+ Tewabe et al.; 2017

Odds ratio $[95 \% \mathrm{Cl}]$

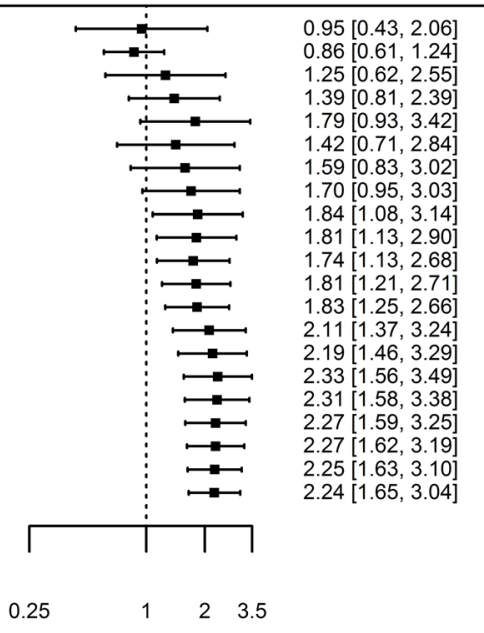

Odds Ratio (log scale)

Figure 10 Forest plot showing the results from a cumulative meta-analysis of studies examining the effect of ANC on EBF. ANC, antenatal care; EBF, exclusive breast feeding.

as a result, it is not relevant to investigate the possible reasons for heterogeneity.

Among studies reporting the association between EBF and gender of newborn, ANC and PNC, $77.66 \%, 60.29 \%$ and $100 \%$ of the heterogeneity were accounted for the variation in study area (region), residence of mothers, sample size and publication year, respectively. Based on the omnibus test of moderators, study area (region) and publication year negatively influenced the association between gender of newborn and EBF practice $\left(Q_{\mathrm{M}}=18.46, \mathrm{df}=7, \mathrm{p}=0.01\right)$. Study area (region) negatively influenced the association between ANC and EBF practice $\left(\mathrm{Q}_{\mathrm{M}}=27.55, \mathrm{df}=8, \mathrm{p}=0.001\right)$ (table 3$)$.

Odds ratio $[95 \% \mathrm{Cl}]$

Abera; 2012
+ Sonko et al; 2015
+ Teka et al; 2015
+ Asemahagn; 2016
+ Tadesse et al; 2016
+ Tewabe et al.; 2017
1

Figure 11 Forest plot showing the results from a cumulative meta-analysis of studies examining the effect of PNC on EBF. EBF, exclusive breast feeding; PNC, postnatal care.

\section{DISCUSSION}

This meta-analysis assessed the association between breastfeeding practices (ie, TIBF and EBF) and gender of newborn, ANC and PNC. The key findings were EBF was significantly associated with ANC, PNC and gender of newborn whereas TIBF was significantly associated with ANC but not with gender of newborn.

In congruent with our hypothesis and the large body of global evidence, ${ }^{61-66}$ our finding indicated that mothers who had at least one antenatal visit had a significantly higher chance of initiating breast feeding within 1 hour of birth and exclusively breast feed for the first 6 months compared with mothers who had no ANC visit. This may be because health professionals provide breastfeeding guidance and counselling during ANC visit. ${ }^{7}$ The Ethiopian Ministry of Health has also adopted Baby-Friendly Hospital Initiative programme as part of the national nutrition programme and is now actively working to integrate to all public and private health facilities and improving breastfeeding practice as a result.

We also showed that mothers who had at least one PNC visit had nearly twice higher chance of exclusively breast feeding during the first 6 months compared with mothers who had no PNC follow-up. This result supported our hypothesis, and various studies have similarly reported a significantly high rate of EBF in mothers who had a postnatal visit at health institution ${ }^{66}$ or postnatal home visit. ${ }^{67}$ The possible justification could be that postnatal visit health education may positively influence the belief and decision of the mothers to exclusively breast feed. Previous studies have also shown that postnatal education and counselling are important to increase EBF practice. ${ }^{68}$ In addition, in our previous meta-analyses, we showed that guidance and counselling during PNC was significantly associated with high-rate EBF. ${ }^{7}$ Furthermore, PNC may ease breastfeeding difficulty, increase maternal confidence and encourage social/family support which lead the mother to continue EBF for 6 months.

Finally, in agreement with our hypothesis and previous studies ${ }^{69-71}$ we uncovered gender of newborn was significantly associated with EBF practice. Mothers with male newborn had a $31 \%$ significantly higher chance of exclusively breast feeding during the first 6 months compared with mothers of female newborn. This finding disproved the traditional perception and belief in Ethiopia that male newborn has prelacteal feeding to be strong and healthy compared with female newborn. On the other hand, several studies ${ }^{6366}$ depicted that gender of newborn is not significantly associated with breastfeeding practice, such as TIBF as we showed in our meta-analysis. This discrepancy might be due to the sociocultural difference and lack of adequate power given that we only found 10 studies to estimate the pooled effect size.

This systematic review and meta-analysis was conducted based on published protocol, ${ }^{15}$ and PRISMA guideline for literature reviews. In addition, publication bias was quantified using Egger's regression statistical test and NOS was used to assess the quality of included studies. Since it is 
Table 3 Meta-regression analysis to identify possible factors of heterogeneity among the included studies

\begin{tabular}{|c|c|c|c|c|c|c|}
\hline Variables (reference category)* & Estimate & SE & $Z$ value & $P$ value & Cl.Ib & Cl.ub \\
\hline \multicolumn{7}{|l|}{ TIBF } \\
\hline \multicolumn{7}{|l|}{ ANC } \\
\hline Amhara region (Afar) & 1.71 & 1.17 & 1.46 & 0.15 & -0.59 & 4.01 \\
\hline Oromia region (Afar) & 1.48 & 0.91 & 1.62 & 0.10 & -0.31 & 3.28 \\
\hline SNNPR region (Afar) & 0.54 & 1.09 & 0.50 & 0.62 & -1.58 & 2.67 \\
\hline Tigray region (Afar) & 1.58 & 1.30 & 1.21 & 0.23 & -0.97 & 4.12 \\
\hline Urban residence (Rural) & 0.71 & 1.07 & 0.67 & 0.51 & -1.38 & 2.80 \\
\hline Urban and rural residence (Rural) & 0.65 & 1.25 & 0.52 & 0.61 & -1.81 & 3.10 \\
\hline$\geq 501$ mothers ( $\leq 500$ mothers) & -0.54 & 0.81 & -0.66 & 0.51 & -2.13 & 1.06 \\
\hline Published 2016-2018 (2011-2015) & 0.14 & 0.82 & 0.17 & 0.87 & -1.47 & 1.74 \\
\hline
\end{tabular}

EBF

Gender of newborn

\begin{tabular}{|c|c|c|c|c|c|c|}
\hline Oromia region (Amhara) & -0.54 & 0.24 & -2.22 & 0.03 & -1.02 & -0.06 \\
\hline SNNPR region (Amhara) & 0.12 & 0.26 & 0.46 & 0.64 & -0.39 & 0.63 \\
\hline Tigray region (Amhara) & -0.39 & 0.30 & -1.31 & 0.19 & -0.98 & 0.19 \\
\hline Urban residence (Rural) & 0.79 & 0.51 & 1.57 & 0.12 & -0.20 & 1.78 \\
\hline Urban and rural residence (Rural) & -0.10 & 0.44 & -0.24 & 0.81 & -0.96 & 0.75 \\
\hline$\geq 501$ mothers ( $\leq 500$ mothers) & 0.78 & 0.23 & 3.34 & $<0.001$ & 0.32 & 1.24 \\
\hline Published 2016-2018 (2011-2015) & -1.14 & 0.44 & -2.59 & 0.01 & -1.99 & -0.28 \\
\hline \multicolumn{7}{|l|}{ ANC } \\
\hline Harari region (Amhara) & -0.11 & 0.64 & -0.17 & 0.87 & -1.37 & 1.16 \\
\hline Oromia region (Amhara) & -1.27 & 0.39 & -3.28 & 0.001 & -2.03 & -0.51 \\
\hline SNNPR region (Amhara) & 0.09 & 0.35 & 0.27 & 0.78 & -0.59 & 0.78 \\
\hline Tigray region (Amhara) & -0.49 & 0.57 & -0.87 & 0.38 & -1.60 & 0.62 \\
\hline Urban residence (Rural) & -0.18 & 0.38 & -0.47 & 0.63 & -0.92 & 0.56 \\
\hline Urban and rural residence (Rural) & -0.26 & 0.52 & -0.49 & 0.62 & -1.28 & 0.76 \\
\hline$\geq 501$ mothers ( $\leq 500$ mothers) & -0.30 & 0.34 & -0.87 & 0.38 & -0.96 & 0.37 \\
\hline Published 2016-2018 (2011-2015) & 0.08 & 0.28 & 0.29 & 0.77 & -0.46 & 0.62 \\
\hline \multicolumn{7}{|l|}{ PNC† } \\
\hline Harari region (Amhara) & -0.60 & 0.48 & -1.24 & 0.22 & -1.54 & 0.35 \\
\hline SNNPR region (Amhara) & 0.25 & 0.30 & 0.82 & 0.41 & -0.34 & 0.83 \\
\hline Tigray region (Amhara) & -0.16 & 0.64 & -0.25 & 0.80 & -1.42 & 1.10 \\
\hline$\geq 501$ mothers ( $\leq 500$ mothers) & 0.11 & 0.31 & 0.36 & 0.72 & -0.50 & 0.73 \\
\hline Published 2016-2018 (2011-2015) & 0.26 & 0.36 & 0.71 & 0.47 & -0.45 & 0.96 \\
\hline
\end{tabular}

*Since we do not have a specific hypothesis, the reference category is selected arbitrarily; †Residence is dropped from the model due to small sample size of included studies. Cut-off value for sample size and publication year was arbitrarily chosen.

ANC, antenatal care; Cl.Ib, Clinterval, lower bound; Cl.ub, Cl interval, upper bound; EBF, exclusive breast feeding; PNC, postnatal care;

SNNPR, Southern Nations, Nationalities and Peoples' Region; TIBF, timely initiation of breast feeding.

the first study in Ethiopia, the evidence could be helpful for future researchers, public health practitioners and healthcare policy-makers. The inclusion of all previously published studies is a further strength of this meta-analysis. This study has limitations as well. Almost all included studies were observational, which weakens the strength of evidence and hinder causality inference. Even though we have used broad search strategies, the possibility of missing relevant studies cannot be fully exempted and the finding may not be nationally representative. Based on the conventional method of heterogeneity test, a few analyses suffer from high between-study variation. The course of heterogeneity was carefully explored using meta-regression analysis, and this variation may be due to the difference in study area (region), residence of mothers, sample size, publication year or other residual 
factors; therefore, the result should be interpreted with caution. Moreover, the dose-response relationship between the number of ANC or PNC visits and breastfeeding practices was not examined. Lastly, significant publication bias was detected in studies that reported the association between EBF and gender of newborn. We did Duval and Tweedie trim-and-fill analysis to adjust publication bias and to provide an unbiased estimate; however, the result should be cautiously interpreted.

\section{CONCLUSIONS}

In line with our hypothesis, we found that increasing the use of antenatal and PNC has a positive effect on breastfeeding practices (ie, TIBF and EBF), which signifies stakeholders would provide emphasis on ANC and PNC service to optimise breast feeding. This meta-analysis study provided an overview of up-to-date evidence for public nutrition professionals and policy-makers in Ethiopia. It could also be useful for breastfeeding improvement initiative in Ethiopia and cross-country and cross-cultural comparison. From the research point of view, in general, intervention and outcome based studies on breast feeding in Ethiopia are required.

\section{Author affiliations}

${ }^{1}$ Department of Nursing, Debre Berhan University, Debre Berhan, Ethiopia ${ }^{2}$ Department of Epidemiology, University Medical Center Groningen, University of Groningen, Groningen, The Netherlands

${ }^{3}$ Department of Public Health, University Medical Center Groningen, University of Groningen, Groningen, The Netherlands

Acknowledgements Our special gratitude forwarded to Sjoukje van der Werf (University of Groningen, the Netherlands) for her support to develop the search strings and Balewgizie Sileshi (University of Groningen, the Netherlands) for his support during the title and abstract screening.

Contributors NTS and TDH conceived and designed the study. TDH developed a syntax for searching databases, analysed the data and interpreted the results. TDH and SMA wrote and revised the manuscript. All authors read and approved the final manuscript.

Funding The authors have not declared a specific grant for this research from any funding agency in the public, commercial or not-for-profit sectors.

Competing interests None declared.

Patient consent for publication Not required.

Provenance and peer review Not commissioned; externally peer reviewed.

Data sharing statement All data generated or analysed in this study are included in the article and its supplementary files.

Open access This is an open access article distributed in accordance with the Creative Commons Attribution Non Commercial (CC BY-NC 4.0) license, which permits others to distribute, remix, adapt, build upon this work non-commercially, and license their derivative works on different terms, provided the original work is properly cited, appropriate credit is given, any changes made indicated, and the use is non-commercial. See: http://creativecommons.org/licenses/by-nc/4.0/.

\section{REFERENCES}

1. World Health Organization. Infant and young child feeding: a tool for assessing national practices, policies and programmes, 2003.

2. World Health Organization. Babies and mothers worldwide failed by lack of investment in breastfeeding. Saudi Med J 2017;38:974-5.
3. Edmond KM, Zandoh C, Quigley MA, et al. Delayed breastfeeding initiation increases risk of neonatal mortality. Pediatrics 2006;117:e380-6.

4. World Health Organization. Infant and young child feeding: model chapter for textbooks for medical students and allied health professionals. Infant and young child feeding: model chapter for textbooks for medical students and allied health professionals, 2009.

5. Unicef W. Capture the Moment - Early initiation of breastfeeding: The best start for every newborn, 2018.

6. UNICEF. Infant and young child feeding. 2018. Available at https:// data.unicef.org/topic/nutrition/infant-and-young-child-feeding/ (Accessed 20 Sep 2018).

7. Habtewold TD, Mohammed SH, Endalamaw A, et al. Breast and complementary feeding in Ethiopia: new national evidence from systematic review and meta-analyses of studies in the past 10 years. Eur J Nutr 2018.

8. Boccolini CS, MLd C. Oliveira, Maria Inês Couto de. Factors associated with exclusive breastfeeding in the first six months of life in Brazil: a systematic review. Rev Saude Publica 2015:49.

9. Sharma IK, Byrne A. Early initiation of breastfeeding: a systematic literature review of factors and barriers in South Asia. Int Breastfeed $J$ 2016;11:17.

10. Alemu SM, Alemu YM, Habtewold TD. Association of age and colostrum discarding with breast-feeding practice in Ethiopia: systematic review and meta-analyses. Public Health Nutr 2019:1-20.

11. Alebel A, Dejenu G, Mullu G, et al. Timely initiation of breastfeeding and its association with birth place in Ethiopia: a systematic review and meta-analysis. Int Breastfeed J 2017;12:44.

12. Alebel A, Tesma C, Temesgen B, et al. Exclusive breastfeeding practice in Ethiopia and its association with antenatal care and institutional delivery: a systematic review and meta-analysis. Int Breastfeed J 2018:13:31.

13. Temesgen $\mathrm{H}$, Negesse A, Woyraw $\mathrm{W}$, et al. Dietary diversity feeding practice and its associated factors among children age 6-23 months in Ethiopia from 2011 up to 2018: a systematic review and metaanalysis. Ital J Pediatr 2018;44:109.

14. Temesgen $\mathrm{H}$, Negesse A, Woyraw W, et al. Prelacteal feeding and associated factors in Ethiopia: systematic review and meta-analysis. Int Breastfeed J 2018;13:49.

15. Habtewold TD, Islam MA, Sharew NT, et al. SystEmatic review and meta-aNAlysis of infanT and young child feeding Practices (ENAT-P) in Ethiopia: protocol. BMJ Open 2017;7:e017437.

16. Kraemer HC. Kappa coefficient. Wiley StatsRef: Statistics Reference Online 2014:1-4.

17. Peterson J, Welch V, Losos M, et al. The Newcastle-Ottawa scale (NOS) for assessing the quality of nonrandomised studies in metaanalyses. 2011.

18. Munn Z, Tufanaru C, Aromataris E. JBI's systematic reviews: data extraction and synthesis. Am J Nurs 2014;114:49-54.

19. Brinkhoff T. Federal Democratic Republic of Ethiopia. 2015. 2018. Available at http://www.citypopulation.de/Ethiopia.html.

20. Egger M, Davey Smith G, Schneider M, et al. Bias in meta-analysis detected by a simple, graphical test. BMJ 1997;315:629-34.

21. Duval S, Tweedie R. Trim and fill: A simple funnel-plot-based method of testing and adjusting for publication bias in meta-analysis. Biometrics 2000;56:455-63.

22. Higgins JP, Thompson SG. Quantifying heterogeneity in a metaanalysis. Stat Med 2002;21:1539-58.

23. Viechtbauer W. Conducting Meta-Analyses in $R$ with the metafor Package. J Stat Softw 2010;36.

24. Moher D, Liberati A, Tetzlaff J, et al. Preferred reporting items for systematic reviews and meta-analyses: the PRISMA statement. PLoS Med 2009;6:e1000097.

25. Regassa N. Infant and child feeding practices among farming communities in Southern Ethiopia. Kontakt 2014;16:e215-22.

26. Alemayehu M. Factors Associated with Timely Initiation and Exclusive Breast Feeding among Mothers of Axum Town, Northern Ethiopia. Science Journal of Public Health 2014;2:394-401.

27. Berhe H, Mekonnen B, Bayray A, et al. Determinants of Breast feeding Practices Among Mothers Attending Public Health Facilities, Mekelle, Northern Ethiopia; A Cross Sectional Study. International Journal of Pharmaceutical Sciences and Research 2013;4:650.

28. Beyene MG, Geda NR, Habtewold TD, et al. Early initiation of breastfeeding among mothers of children under the age of 24 months in Southern Ethiopia. Int Breastfeed J 2016;12.

29. Lakew Y, Tabar L, Haile D. Socio-medical determinants of timely breastfeeding initiation in Ethiopia: Evidence from the 2011 nation wide Demographic and Health Survey. Int Breastfeed J 2015;10. 
30. Liben ML, Yesuf EM. Determinants of early initiation of breastfeeding in Amibara district, Northeastern Ethiopia: a community based crosssectional study. Int Breastfeed J 2016;11.

31. Setegn T, Gerbaba M, Belachew T. Determinants of timely initiation of breastfeeding among mothers in Goba Woreda, South East Ethiopia: a cross sectional study. BMC Public Health 2011;11:217,2458-11217.

32. Wolde T, Birhanu T, Ejeta E. Prevalence and determinants of timely initiation of breastfeeding among lactating mothers of urban dwellers in Western Ethiopia: A community based cross sectional study. Food Science and Quality Management 2014:31.

33. Woldemichael B. Timely Initiation of Breastfeeding and Its Associated Factors among Mothers in Tiyo Woreda, Arsi Zone, Ethiopia: A Community- Based Cross Sectional Study. Clinics in Mother and Child Health 2016;13:221.

34. Mekonen L, Seifu W, Shiferaw Z. Timely initiation of breastfeeding and associated factors among mothers of infants under 12 months in South Gondar zone, Amhara regional state, Ethiopia; 2013. Int Breastfeed J 2018;13:17.

35. Gultie T, Sebsibie G. Determinants of suboptimal breastfeeding practice in Debre Berhan town, Ethiopia: a cross sectional study. Int Breastfeed J 2016;11

36. Tamiru D, Belachew T, Loha E, et al. Sub-optimal breastfeeding of infants during the first six months and associated factors in rural communities of Jimma Arjo Woreda, Southwest Ethiopia. BMC Public Health 2012;12:363,2458-12-363.

37. Tamiru D, Tamrat M. Constraints to the optimal breastfeeding practices of breastfeeding mothers in the rural communities of Arba Minch Zuria Woreda, Ethiopia: a community-based, cross-sectional study. South African Journal of Clinical Nutrition 2015;28:134-9.

38. Adugna DT. Women's perception and risk factors for delayed initiation of breastfeeding in Arba Minch Zuria, Southern Ethiopia. Int Breastfeed J 2014;9.

39. Derso T, Biks GA, Tariku A, et al. Correlates of early neonatal feeding practice in Dabat HDSS site, northwest Ethiopia. Int Breastfeed J 2017;12.

40. Tewabe T. Timely initiation of breastfeeding and associated factors among mothers in Motta town, East Gojjam zone, Amhara regional state, Ethiopia, 2015: a cross-sectional study. BMC Pregnancy Childbirth 2016;16:314.

41. Musa Seid A. Vaginal Delivery and Maternal Knowledge on Correct Breastfeeding Initiation Time as Predictors of Early Breastfeeding Initiation: Lesson from a Community-Based Cross-Sectional Study. ISRN Epidemiology 2014;2014:1-6.

42. Asemahagn MA. Determinants of exclusive breastfeeding practices among mothers in azezo district, northwest Ethiopia. Int Breastfeed $J$ $2016 ; 11$.

43. Setegn T, Belachew T, Gerbaba M, et al. Factors associated with exclusive breastfeeding practices among mothers in Goba district, south east Ethiopia: A cross-sectional study. Int Breastfeeding J 2012:7.

44. Sonko A, Worku A. Prevalence and predictors of exclusive breastfeeding for the first six months of life among women in Halaba special woreda, Southern Nations, Nationalities and Peoples' Region/SNNPR/, Ethiopia: a community based cross-sectional study. Arch Public Health 2015;73.

45. Biks GA, Tariku A, Tessema GA. Effects of antenatal care and institutional delivery on exclusive breastfeeding practice in northwest Ethiopia: a nested case-control study. Int Breastfeed J 2015;10:30.

46. Arage G, Gedamu H. Exclusive Breastfeeding Practice and Its Associated Factors among Mothers of Infants Less Than Six Months of Age in Debre Tabor Town, Northwest Ethiopia: A Cross-Sectional Study. Adv Public Health 2016;2016:1-7.

47. Adugna B, Tadele H, Reta F, et al. Determinants of exclusive breastfeeding in infants less than six months of age in Hawassa, an urban setting, Ethiopia. Int Breastfeed J 2017;12:45.

48. Egata G, Berhane Y, Worku A. Predictors of non-exclusive breastfeeding at 6 months among rural mothers in east Ethiopia: a community-based analytical cross-sectional study. Int Breastfeed $J$ 2013;8:8.

49. Teka B, Assefa H, Haileslassie K. Prevalence and determinant factors of exclusive breastfeeding practices among mothers in Enderta woreda, Tigray, North Ethiopia: a cross-sectional study. Int Breastfeed J 2015;10:2

50. Sefene A. Determinants of Exclusive Breastfeeding Practice among Mothers of Children Age Less Than 6 Month in Bahir Dar
City Administration, Northwest Ethiopia; A Community Based Cross-Sectional Survey. Science Journal of Clinical Medicine 2013;2:153-9.

51. Seid AM, Yesuf ME, Koye DN. Prevalence of Exclusive Breastfeeding Practices and associated factors among mothers in Bahir Dar city, Northwest Ethiopia: a community based cross-sectional study. Int Breastfeed J 2013;8:14.

52. Hunegnaw MT, Gezie LD, Teferra AS. Exclusive breastfeeding and associated factors among mothers in Gozamin district, northwest Ethiopia: a community based cross-sectional study. Int Breastfeed J $2017 ; 12$.

53. Lenja A, Demissie T, Yohannes B, et al. Determinants of exclusive breastfeeding practice to infants aged less than six months in Offa district, Southern Ethiopia: a cross-sectional study. Int Breastfeed J 2016;11:32.

54. Tadesse T, Mesfin F, Chane T. Prevalence and associated factors of non-exclusive breastfeeding of infants during the first six months in rural area of Sorro District, Southern Ethiopia: a cross-sectional study. Int Breastfeed J 2016;11. 25,016-0085-6. eCollection 2016.

55. Tariku A, Alemu K, Gizaw Z, et al. Mothers' education and ANC visit improved exclusive breastfeeding in Dabat Health and Demographic Surveillance System Site, northwest Ethiopia. PLoS One 2017;12:e0179056.

56. Abera K. Infant and young child feeding practices among mothers living in Harar, Ethiopia. Harar Bulletin of Health Sciences 2012;4:66-78.

57. Taddele M. Exclusive Breastfeeding and Maternal Employment in Ethiopia: A Comparative Cross- Sectional Study. International Journal of Nutrition and Food Sciences 2014;3:497-503.

58. Echamo M. Exclusive breast feeding in Arbaminch, SNNPR, Ethiopia Harar Bull Health Sci 2012;5:44-59.

59. Chekol DA, Biks GA, Gelaw YA, et al. Exclusive breastfeeding and mothers' employment status in Gondar town, Northwest Ethiopia: a comparative cross-sectional study. Int Breastfeed J 2017;12:27.

60. Tewabe T, Mandesh A, Gualu T, et al. Exclusive breastfeeding practice and associated factors among mothers in Motta town, East Gojjam zone, Amhara Regional State, Ethiopia, 2015: a crosssectional study. Int Breastfeed J 2016;12.

61. Patel A, Badhoniya N, Khadse S, et al. South Asia Infant Feeding Research Netwoork. Infant and young child feeding indicators and determinants of poor feeding practices in India: secondary data analysis of National Family Health Survey 2005-06. Food Nutr Bull 2010;31:314-33.

62. Mihrshahi S, Kabir I, Roy SK, et al. South Asia Infant Feeding Research Network. Determinants of infant and young child feeding practices in Bangladesh: secondary data analysis of Demographic and Health Survey 2004. Food Nutr Bull 2010;31:295-313.

63. Senarath U, Dibley MJ, Godakandage SS, et al. South Asia Infant Feeding Research Network (SAIFRN)*. Determinants of infant and young child feeding practices in Sri Lanka: secondary data analysis of Demographic and Health Survey 2000. Food and nutrition bulletin 2010;31:352-65

64. Ogunlesi TA. Maternal socio-demographic factors influencing the initiation and exclusivity of breastfeeding in a Nigerian semi-urban setting. Matern Child Health J 2010;14:459-65.

65. Okafor IP, Olatona FA, Olufemi OA. Breastfeeding practices of mothers of young children in Lagos, Nigeria. Niger J Paediatr 2014:41:43-7.

66. Subedi N, Paudel S, Rana T, et al. Infant and young child feeding practices in Chepang communities. J Nepal Health Res Counc 2012;10:141-6.

67. Bashour HN, Kharouf MH, Abdulsalam AA, et al. Effect of postnata home visits on maternal/infant outcomes in Syria: a randomized controlled trial. Public Health Nurs 2008;25:115-25.

68. Su LL, Chong YS, Chan YH, et al. Antenatal education and postnatal support strategies for improving rates of exclusive breast feeding: randomised controlled trial. BMJ 2007;335:596.

69. Agho KE, Dibley MJ, Odiase JI, et al. Determinants of exclusive breastfeeding in Nigeria. BMC Pregnancy Childbirth 2011;11:2.

70. Ogada IA. Effectiveness of couple counselling versus maternal counselling in promoting exclusive breast feeding: a randomised controlled trial in Nyando District, Kenya. 2014.

71. Al Ghwass MM, Ahmed D. Prevalence and predictors of 6-month exclusive breastfeeding in a rural area in Egypt. Breastfeed Med 2011;6:191-6. 
Correction: Evidence on the effect of gender of new-born, antenatal care and postnatal care on breastfeeding practices in Ethiopia: a meta-analysis and meta-regression analysis of observational studies

Habtewold TD, Sharew NT, Alemu SM. Evidence on the effect of gender of newborn, antenatal care and postnatal care on breastfeeding practices in Ethiopia: a meta-analysis and meta-regression analysis of observational studies. BMJ Open 2019;9:e023956. doi: 10.1136/bmjopen-2018-023956

The following amendments were considered to the original version of this article.

Reference 35: Gultie T, Sebsibie G. Determinants of suboptimal breastfeeding practice in Debre Berhan town, Ethiopia: a cross sectional study. Int Breastfeed J 2016;11 has been excluded from the published article.

Authors have found in their meta-analysis, ${ }^{1}$ that this study in reference $35^{2}$ was retracted from the International Breastfeeding Journal in 2018 (online: 07 March 2018) because of significant overlap of both text and data with the Master's Thesis of Hilina Ketma, "Assessment of prevalence and determinants of suboptimal breastfeeding among mothers of children aged less than two years in Dire Dawa City Administration, Ethiopia, June 2013", which was defended at the School of Graduate Studies, Addis Ababa University, Addis Ababa, Ethiopia in June 2013. ${ }^{3}$

Therefore, authors have performed reanalysis by excluding Gultie and Sebsibie study (reference 35), and revised figure 3 and figure 5 .

In conclusion, despite having excluded Gultie and Sebsibie study, the results show that antenatal care significantly associated with timely initiation of breastfeeding and exclusive breastfeeding. Therefore, the central findings of the original article remain unaffected.

Please, find the revised figures.

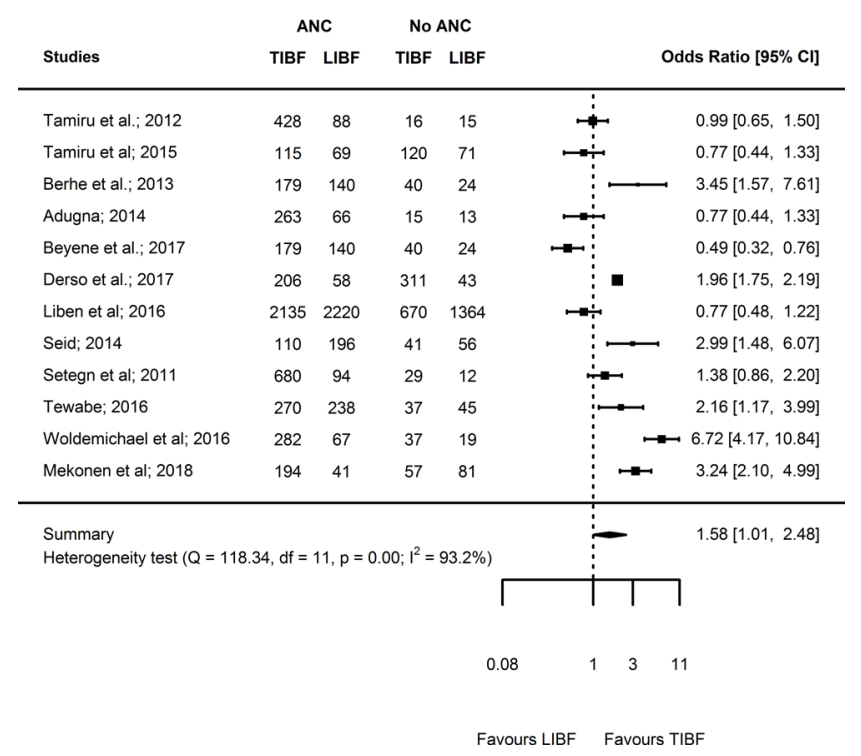

Figure 3 Forest plot of the unadjusted odds ratios with corresponding $95 \%$ cis of 13 studies on the association of ANC and TIBF. The horizontal line represents the $\mathrm{Cl}$, the box and its size in the middle of the horizontal line represents the weight of sample size. The polygon represents the pooled or. The reference category is 'no ANC follow-up'. ANC, antenatal care; LIBF, late initiation of breast feeding; REM, random-effects model; TIBF, timely initiation of breast feeding. 


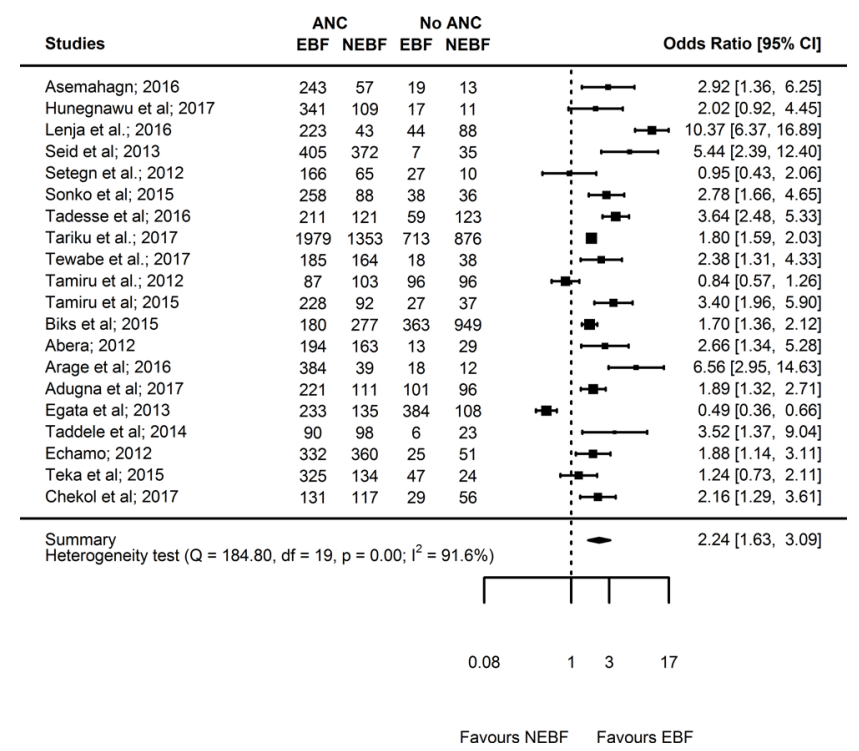

Figure 5 Forest plot of the unadjusted odds ratios with corresponding $95 \%$ cis of 21 studies on the association of ANC and EBF. The horizontal line represents the $\mathrm{Cl}$, the box and its size in the middle of the horizontal line represents the weight of sample size. The polygon represents the pooled or. The reference category is 'no ANC follow-up'. ANC, antenatal care; EBF, exclusive breast feeding; NEBF, non-exclusive of breast feeding; REM, random-effects model.

\section{REFERENCES}

1 Habtewold TD, Sharew NT, Alemu SM. Evidence on the effect of gender of newborn, antenatal care and postnatal care on breastfeeding practices in Ethiopia: a meta-analysis and meta-regression analysis of observational studies. BMJ Open 2019;9:e023956.

2 Gultie T, Sebsibie G. Determinants of suboptimal breastfeeding practice in Debre Berhan town, Ethiopia: a cross sectional study. Int Breastfeed J 2016;11:5. eCollection 2016.

3 Gultie T, Sebsibie G. Retraction note: determinants of suboptimal breastfeeding practice in Debre Berhan town, Ethiopia: a cross sectional study. Int Breastfeed J2018;13:13.

Open access This is an open access article distributed in accordance with the Creative Commons Attribution Non Commercial (CC BY-NC 4.0) license, which permits others to distribute, remix, adapt, build upon this work non-commercially, and license their derivative works on different terms, provided the original work is properly cited, appropriate credit is given, any changes made indicated, and the use is non-commercial. See: http://creativecommons.org/licenses/by-nc/4.0/.

(C) Author(s) (or their employer(s)) 2020. Re-use permitted under CC BY-NC. No commercial re-use. See rights and permissions. Published by BMJ.

BMJ Open 2020;10:e23956corr1. doi:10.1136/bmjopen-2018-023956corr1

A) Check for updates 